\title{
Dr. Google-Diät überwinden
}

E ltern sind oft überzeugt, dass hinter - atopischer Dermatitis (AD) oder anderen allergischen Erkrankungen ihrer Kinder eine Nahrungsmittelallergie steckt. Internetinformationen bestärken sie, ihren Kindern auch ohne ärztliche Beratung unsinnige und strikte Diäten aufzuerlegen.

„Bei Dr. Goggle“ findet sich gefährliches Halbwissen, werden Begriffe vermischt, Altersgruppen nicht auseinander gehalten und teils veraltete Informationen geliefert", stellte Kinderärztin Dr. Ute Staden, Berlin, bei der eigenen Recherche zur AD fest. Ob „Eatsmarter“, „Netdoktor“ oder „Reformhaus Ratgeber“, bei Neurodermitis wird die Diät unkritisch als wesentliche Behandlung empfohlen. „Dabei haben zwei Drittel der Kinder mit AD gar keine Nahrungsmittelallergie“, betonte sie.

Die Nahrungsmittelprovokation kann die tatsächliche Allergie aufklären und eine differenziertere Therapie ermöglichen. So im Falle eines zehn Monate alten Jungen, der im 5. Monat die Prä-
Nahrung verweigerte, im 7. Lebensmonat nach zwei Löffel Kuhmilchbrei eine periorale Rötung und Quaddeln, im 8. Monat bereits nach einem Löffel Kuhmilchbrei eine starke Lippenschwellung entwickelte und im 9. Lebensmonat eine infektassoziierte obstruktive Bronchitis hatte. Der betreuende Kinderarzt fand in der serologischen Diagnostik keinen Hinweis auf eine Allergie. Der von Dr. Staden selbst durchgeführte Pricktest im 10. Monat zeigte einen Hautindex auf Kuhmilch von 2,3. Sie riet, erst einmal soweit möglich - weiter zu stillen und mittelfristig einen Provokationstest durchzuführen. Bei der Wiedervorstellung sechs Monate später vertrug der Junge verbackenes Ei, bei kleinen Mengen von Rührei traten wieder periorale Rötung und Quaddeln auf. Der serologische Test fiel für Kuhmilch/Kasein negativ aus, aber Hühnereiweis und das hitzestabile GAL d1 waren leicht erhöht. Im Pricktest lag der Kuhmilchhautindex nur noch bei 1,3, der Index für Hühnereiweiß bei 1,7. Weil der Besuch der Kin- dertagesstätte anstand, waren die Eltern schließlich zu einer stationären Nahrungsmittelprovokation bereit. Dort reagierte das Kind auf Kuhmilch nicht, aber auf rohes Hühnerei, allerdings erst 2,5 Stunden nach der letzten Gabe, dann aber heftig mit generalisierter Rötung, generalisierter Urtikaria sowie deutlicher Obstruktion.

In der Konsequenz darf der Junge weiterhin Milchprodukte verzehren. Verbackenes Hühnerei, das er zuvor vertragen hatte, bekommt er aber zukünftig nur von den Eltern. „In der KiTa machen wir da keine Experimente“, betonte Staden. Aufgrund der schweren Reaktion im Provokationstest hat sie eine Schulung durchgeführt und ein Notfallset mit Adrenalinpen verschrieben. „Dank der Nahrungsmittelprovokation fällt nur ein Nahrungsmittel weg, ansonsten kann das Kind normal ernährt werden“, resümierte sie.

Friederike Klein

Allergo-Compact: „Zwischen Unverträglichkeit und Auslassdiät als Trend der Zeit"

einer Penicillinallergie bedeutsam sei, weil dies im weiteren Lebensverlauf notwendig werdende Antibiotikatherapien erleichtert.

Kathrin von Kieseritzky eine kontrollierte Provokationstestung erfolgen. In bis zu $95 \%$ der Fälle falle dann auch der Provokationstest negativ aus, so Trautmann. Doch $100 \%$ ige Sicherheit biete nur die kontrollierte Provokation. Bei Hinweisen auf schwere Reaktionen sollte auf eine ambulante Provokation verzichtet werden, die Patienten sind am besten in ein entsprechend spezialisiertes Zentrum zu überweisen. Erweisen sich die Provokationstests als positiv, ist die Allergie damit eindeutig nachgewiesen, sind sie negativ, kann auch die Allergie klar ausgeschlossen werden.

Eine Allergiediagnostik kann das auslösende Penicillin oder Cephalosporin mit Sicherheit identifizieren. Das Allergen bilden in der Regel die AminobenzylSeitenketten, die bei den Cefalosporinen der neueren Generationen verändert worden sind, weshalb diese Mittel oft vertragen werden. Trautmann verwies zudem darauf, dass auch der Ausschluss
Curriculum der Allergologie: „Moderne Diagnostik von ..."

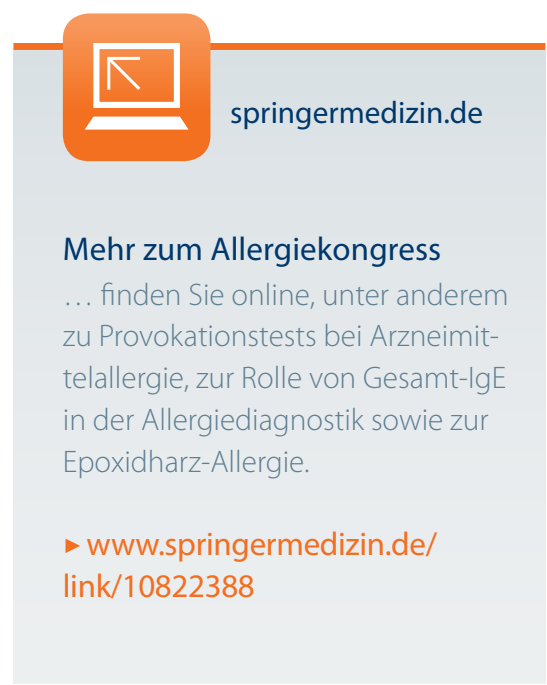

